\title{
VERY DEEP SURVEYS
}

\author{
Y. TANIGUCHI \\ Astronomical Institute, Tohoku University \\ Aramaki, Aoba, Sendai 980-77, Japan
}

\section{Introduction}

When and how did galaxies form ? In order to understand this important problem in astrophysics, many observational studies have been conducted so far. Deep survey observations in the optical are powerful for detecting very faint sources in the high- $z$ universe The observed surface density of such high- $z$ star forming galaxies may, however, be smaller than the value expected from the local density of luminous galaxies (e.g., Lanzetta et al. 1996; Steidel et al. 1996) and it is possible that we are missing classes of objects with substantial reddening and have not yet sampled fully the high- $z$ counterparts of typical nearby galaxies. Since the nearby, well evolved galaxies consist of old low-mass stars, it is important to find galaxies with numerous low-mass stars. These stars emit their energy from the optical to near infrared (NIR) mostly, implying that they are more luminous in the NIR and MIR (mid-infrared) than in the optical if they were present at high redshifts.

Since there are many massive elliptical galaxies in the local universe, it is very important to find their high- $z$ counterparts. The standard galactic wind model for elliptical galaxies (Arimoto and Yoshii 1987) suggests that the forming phase before the galactic winds could be heavily obscured by abundant gas and dust inside the system. If these galaxies were commonly present at high redshifts, they can never been found by the existing deep optical and NIR surveys because of the heavy extinction in the rest-frame ultraviolet and optical wavelength regions (Taniguchi et al. 1994). However, if we perform a very deep survey in the MIR, it is possible to detect such high-z reddened populations. At present, only ISO (Kessler et al.' 1996) allows us to perform such a deep survey since ISOCAM has a very high sensitivity in the MIR (Cesarsky et al. 1996).

\section{Very deep surveys in the mid infrared}

A couple of very deep MIR surveys have been made using ISOCAM (Rowan-Robinson et al. 1997; Taniguchi et al. 1997). A brief summary of the two surveys is given in Table 1. Rowan-Robinson et al. (1997) made the ISOCAM observations of the Hubble Deep Field (HDF, Williams et al. 1996) at 7 and $15 \mu \mathrm{m}$. Combined with the optical multi-color photometry, these MIR data have been used to investigate the star formation properties of galaxies. Rowan-Robinson et al. (1997) modeled the spectral energy distribution of $14 \mathrm{HDF}$ galaxies with redshifts from 0.3 to 1.6 and found indeed that 10 galaxies show the strong MIR excess, providing evidence for intense starbursts in these galaxies ${ }^{1}$ (see also Oliver 1997 in this volume).

Another MIR deep survey program has been made by Taniguchi et al. (1997) whose main aim is addressed to the discovery of heavily reddened populations at high redshifts. Their target field is selected in the Lockman HI hole, LH-NW $\left[\alpha(2000)=10^{\mathrm{h}} 33^{\mathrm{m}} 55.5^{\mathrm{B}}\right.$ and $\delta(2000)=57^{\circ} 46^{\prime}$ $18^{\prime \prime}$ ], because the Galactic HI column density, $\sim 4 \times 10^{19} \mathrm{~cm}^{-2}$, is the lowest value found over the entire sky, providing the best cosmological window for any extragalactic deep survey observations (Jahoda, Lockman, and McCammon 1990). The background fluctuation of the central part of the final mosaiced image is $0.09 \mu \mathrm{Jy} \operatorname{arcsec}^{-2}$, corresponding to a $3 \sigma$ detection limit of $\simeq 9.7 \mu \mathrm{Jy}$ beam $^{-1}$ (the beam size $=6^{\prime \prime} \times 6^{\prime \prime}$ ). This limit is very close to the expected value in the ISOCAM

\footnotetext{
${ }^{1}$ Recently Aussel et al. (1997) analyzed the ISO-HDF data independently using the PRETI (Pattern REcognition Technique for ISOCAM) method developed at Saclay.
}

1125

J. Andersen (ed.), Highlights of Astronomy, Volume IIB, 1125-1127.

(C) 1998 IAU. Printed in the Netherlands. 


\section{ISOCAM $7 \mu \mathrm{m}$ image (halftone) and $\mathrm{H}+\mathrm{K}$ image (contour)}

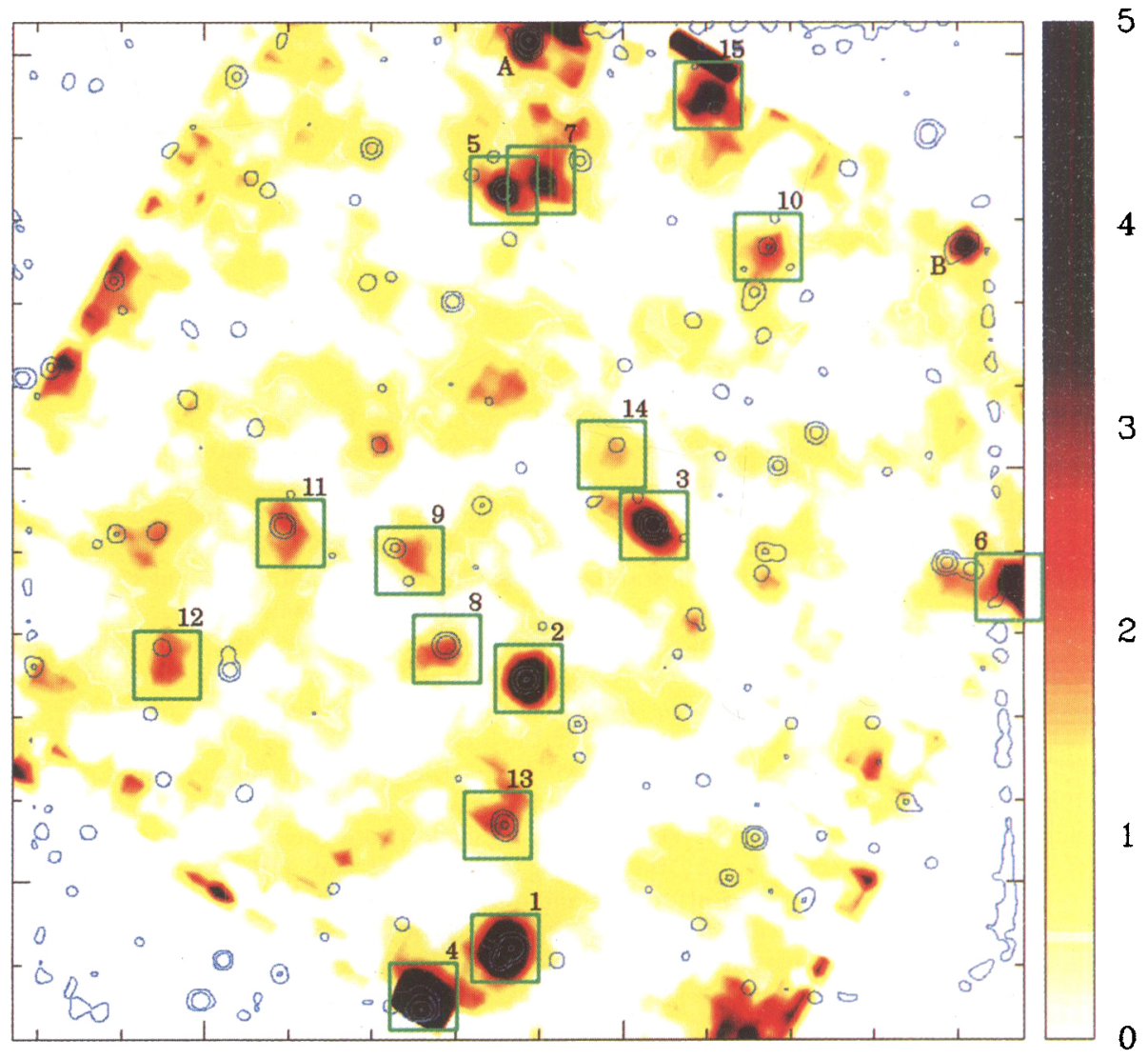

Figure 1. The final $7 \mu \mathrm{m}$ mosaiced image of the LH-NW area (halftone; darker is brighter). The contours show the NIR $(H K)$ image taken with the University of Hawaii $2.2 \mathrm{~m}$ telescope. Although the seeing size in the NIR image is very good ( $F W H M \simeq 0.8$ arcsec), the image is blurred in order to make the comparison between the LW2 and the NIR images easier. The two sources labeled by $A$ and $B$ have NIR counterparts and thus they may be real sources. North is up and east is left.

observers manual. Thus their deep imaging observations have confirmed the very high in-flight performance of ISOCAM. Although they detected 55 sources ( 27 sources are above $5 \sigma_{\text {rms }}$ ), they described the nature of only 15 sources with quite high significance (see Figure 1).

Among the 15 sources, one source is not observed in their NIR observations because the position angle of the LW2 image is shifted by $\sim 30^{\circ}$ from those of the NIR images. Among the remaining 14 sources, twelve sources have NIR counterparts while two objects have no apparent NIR counterpart [the center position is significantly different $\left(\sim 4^{\prime \prime}\right)$ between the LW2 and NIR images]. The latter class of objects may be heavily reddened populations which have not been detected by existing optical and NIR deep surveys.

The observed flux ratios between LW2 and $K^{\prime}\left[R \equiv f_{\nu}(\mathrm{LW} 2) / f_{\nu}\left(K^{\prime}\right)\right]$ scatter from $\sim 0.2$ to 7.4 although most of them are less than $\sim 1$. Since typical late-type dwarf stars show no MIR excess in their rest-frame spectra, the objects with smaller ratios (e.g., $R<0.5$ ) may be Galactic stars or early type galaxies with moderate redshifts. On the other hand, starburst galaxies and active galactic nuclei usually show a MIR excess (e.g., Rowan-Robinson et al. 1997). Therefore the 
objects with the larger ratios may be such galaxies with moderate redshifts. Most of the sources have $K$ magnitudes between 16 and 20 and thus they may have properties similar to the faint NIR galaxies studied by Cowie et al. (1996). If this is the case, the majority of these $7 \mu \mathrm{m}$ sources may be galaxies or active galactic nuclei with $0.2<z<2$ (Cowie et al. 1996).

The most important conclusion is that ISO enabled us to perform the source detection at a level of $\sim 10 \mu \mathrm{Jy}$ at $7 \mu \mathrm{m}$. These MIR deep survey projects have provided a new frontier in the study of star formation history of galaxies at high redshifts.

I would like to thank all the staff of ISO, in particular Martin Kessler, Catherine Cesarsky, and David Elbaz for their useful comments and encouragement during the course of this work. I would like to thank Michael Rowan-Robinson and Seb Oliver for useful comments. Finally I thank my colleagues, Len Cowie, Dave Sanders, Bob Joseph, Gareth Wynn-Williams, Ken Chambers, H. Okuda, K. Kawara, T. Matsumoto, K. Wakamatsu, Y. Sofue, H. Matsuhara, and Yasu Sato.

TABLE 1. Very deep surveys in the mid infrared

\begin{tabular}{lcccc}
\hline & \multicolumn{2}{c}{ Rowan-Robinson et al. } & \multicolumn{2}{c}{ Taniguchi/Cowie et al. } \\
\hline Field & & HDF & LH-NW & SSA 13 \\
Filter & LW2 & LW3 & LW2 & LW2 \\
$\lambda_{\text {center }}(\mu \mathrm{m})$ & 6.75 & 14.5 & 6.75 & 6.75 \\
TDT $^{a}($ hours $)$ & $6.5^{b}$ & 6 & 13.4 & 18 \\
PFOV $^{b}(\operatorname{arcsec})$ & 3 & 6 & 6 & 6 \\
Area $\left(\right.$ arcmin $\left.^{2}\right)$ & 5 & 15 & 9 & 9 \\
$f\left(5 \sigma_{\text {rms }}\right)(\mu \mathrm{Jy})$ & $40^{d}$ & $200^{d}$ & 16 & $-^{e}$ \\
No. of sources & $27^{d}$ & $22^{d}$ & 55 & $-^{e}$ \\
\hline
\end{tabular}

\footnotetext{
Target dedicated time.

${ }^{b}$ Another 6.5-hour integration has been obtained recently

(Rowan-Robinson 1997, private communication).

c Pixel field of view.

${ }^{d}$ Goldschmidt et al. (1996). See also Aussel et al. (1997).

" Not yet reduced.
}

\section{References}

Arimoto, N., and Yoshii, Y. (1987) Chemical and Photometric Properties of a Galactic Wind Model for Elliptical galaxies, $A \& A, 173,23$

Aussel, H., Elbaz, D., Starck, J.L., and Cesarsky C.J. (1997) Observation of the Hubble Deep Field with ISOCAM: Application of the PRETI Method, in Extragalactic Astronomy in the Infrared, eds. G. Mamon, T. Thuan, J. TranhVan (Gif-sur-Yvette: Ed. Frontieres), in press

Cesarsky, C.J., Abergel, A., Agnese, P., et al., (1996) ISOCAM in Flight, $A \& A$, 315, L32

Cowie, L.L., Songaila, A., Hu, E.M., and Cohen, J.G. (1996) New Insight on Galaxy Formation and Evolution From Keck Spectroscopy of the Hawaii Deep Fields, $A J, 112,839$

Goldschmidt, P., Oliver, S., Serjeant, S., et al. (1997) Observations of the Hubble Deep Field with the Infrared Space Observatory - II. Source detection and photometry, MNRAS, 289, 465

Jahoda, K., Lockman, F.J., and McCammon, D. (1990) Galactic H I and the interstellar medium in Ursa Major, $A p J, 354,184$

Kessler, M.F., Steinz, J.A., Anderegg, M.E., et al. (1996) The Infrared Space Observatory (ISO) mission, $A B A, 315$, L27

Lanzetta, K.M., Yahil, A., and Fernandes-Soto, A. (1996) Star-forming Galaxies at Very High Redshifts, Nature, 381, 759

Rowan-Robinson, M., Mann, R.G., Oliver, S.J., et al. (1997) Observations of the Hubble Deep Field with the Infrared Space Observatory - V. Spectral energy distributions, starburst models and star formation history, MNRAS, 289, 490

Steidel, C.S., Giavalisco, M., Pettini, M., Dickinson, M., and Adelberger, K.L. (1996) Spectroscopic Confirmation of a Population of Normal Star-forming Galaxies at Redshifts $z>3, A p J, 462$, L17

Taniguchi Y., Okuda H., Wakamatsu K., et al. (1994) A Search for Primeval Galaxies and Quasars with ISO, in Evolution of the Universe and Its Observational Quest, ed. K. Sato (Universal Academy Press; Tokyo), 545

Taniguchi, Y. Cowie, L.L. Sato, Y. et al. (1997) ISOCAM 7 micron Deep Survey of the Lockman Hole: A Mid-Infrared Search for Primeval Galaxies, $A \& A$, in press

Williams, R. E., Blacker, B., Dickinson, M., et al. (1996) The Hubble Deep Field: Observations, Data Reduction, and Galaxy Photometry, $A J, 112,1335$ 